\title{
Probing the effect of aroma compounds on the hydrodynamic properties of mucin glycoproteins
}

\author{
Vlad Dinu ${ }^{1,2}\left(\right.$ D $\cdot$ Thomas MacCalman $^{1} \cdot \mathrm{Ni} \mathrm{Yang}^{2} \cdot$ Gary G. Adams $^{3} \cdot$ Gleb E. Yakubov $^{1,2} \cdot$ Stephen E. Harding ${ }^{1} \cdot$ \\ Ian D. Fisk ${ }^{2}$
}

Received: 17 August 2020 / Revised: 12 October 2020 / Accepted: 23 October 2020 / Published online: 13 November 2020

(c) The Author(s) 2020

\begin{abstract}
Aroma compounds are diverse low molecular weight organic molecules responsible for the flavour of food, medicines or cosmetics. Natural and artificial aroma compounds are manufactured and used by the industry to enhance the flavour and fragrance of products. While the low concentrations of aroma compounds present in food may leave no effect on the structural integrity of the mucosa, the effect of concentrated aroma volatiles is not well understood. At high concentrations, like those found in some flavoured products such as e-cigarettes, some aroma compounds are suggested to elicit a certain degree of change in the mucin glycoprotein network, depending on their functional group. These effects are particularly associated with carbonyl compounds such as aldehydes and ketones, but also phenols which may interact with mucin and other glycoproteins through other interaction mechanisms. This study demonstrates the formation of such interactions in vitro through the use of molecular hydrodynamics. Sedimentation velocity studies reveal that the strength of the carbonyl compound interaction is influenced by compound hydrophobicity, in which the more reactive short chain compounds show the largest increase in mucin-aroma sedimentation coefficients. By contrast, the presence of groups that increases the steric hindrance of the carbonyl group, such as ketones, produced a milder effect. The interaction effects were further demonstrated for hexanal using size exclusion chromatography light scattering (SEC-MALS) and intrinsic viscosity. In addition, phenolic aroma compounds were identified to reduce the sedimentation coefficient of mucin, which is consistent with interactions in the non-glycosylated mucin region.
\end{abstract}

Keywords Aroma $\cdot$ Mucin $\cdot$ Interactions $\cdot$ Aldehydes $\cdot$ Phenols $\cdot$ Hydrodynamics

\section{Introduction}

Aroma compounds are low molecular weight compounds which are responsible for the perception of flavour. There have been over 7000 aroma compounds identified in 2014

Special Issue: Analytical Ultracentrifugation 2019.

Vlad Dinu

vlad.dinu@nottingham.ac.uk

Thomas MacCalman

Thomas.MacCalman1@nottingham.ac.uk

Ni Yang

Ni.Yang@nottingham.ac.uk

Gary G. Adams

Gary.adams@nottingham.ac.uk

Gleb E. Yakubov

Gleb.Yakubov@nottingham.ac.uk

Stephen E. Harding

Steve.Harding@ nottingham.ac.uk
Ian D. Fisk

Ian.Fisk@nottingham.ac.uk

1 National Centre for Macromolecular Hydrodynamics, School of Biosciences, University of Nottingham, Sutton Bonington Campus, Leicestershire, UK

2 Division of Food, Nutrition and Dietetics, School of Biosciences, University of Nottingham, Sutton Bonington Campus, Leicestershire, UK

3 International Diabetes Education and Research, Faculty of Medicine and Health Sciences, University of Nottingham, Nottingham, UK 
(Parker et al. 2015). Common classes of aroma compounds include aldehydes, ketones, esters, phenols, thiols, lactones and different cyclic configurations. Fruit, vegetables, processed food and most confectionery products contain very low concentrations of aroma compounds (less than $0.01 \mathrm{mg} /$ $\mathrm{ml}$ or $10 \mathrm{ppm}$ ), most of which have developed naturally during product development, such as thermal treatment or fermentation. However, some products are by design enriched with aroma compounds, to enhance their flavour. The aroma ingredients added to improve the flavour of food and other consumer products are generally recognized as safe (GRAS), and are approved for use in food, pharmaceutics and cosmetics. However, recent applications of flavour technologies led to a significant variation in the concentrations of aroma compounds in a range of products (Dinu et al. 2020). Electronic cigarettes are a prime example of a product in which the concentration of aroma compounds can exceed $5 \%$ of the entire formulation $(50,000 \mathrm{ppm})$, which is approximately 10,000 times higher than the typical concentration of aroma compounds found in fruit. These concentrations are close to or even higher than it is deemed safe for consumption by the Flavor and Extract Manufacturers Association (FEMA) (FEMA FEMA, 2020a; Tierney et al. 2016; Cohen et al. 2020). Therefore, their use in e-cigarettes challenges these fundamental GRAS assumptions, because of the high concentration of aldehyde, ketone and other classes of flavourings in direct contact with the oral and respiratory mucosa.

Irrespective of the mode of administration of flavour compounds (ingestion or inhalation), their effect on the intestinal or respiratory mucosae will be determined by a similar type of interaction mechanism, varying according to the structure and function of different mucins (Linden et al. 2008). Thermodynamic and kinetic mechanisms of interaction are influenced the physical and chemical properties of each type of aroma compound (Parker et al. 2015). Most of them are highly hydrophobic and have a low solubility in water, but the shorter chain compounds are also soluble in aqueous solvents. In addition, each molecule has a different freezing and boiling point which would have a significant effect on the properties of the mucus surface, such as density and viscosity effects, but also on the properties of other biological systems in contact with the aroma compounds.

Interactions between aroma compounds and proteins have been extensively reported in the past few decades (Fares et al. 1998; Jouenne and Crouzet 2000; Paravisini and Guichard 2016; Dinu et al. 2019b). They are generally grouped into three types: (i) binding of flavour compounds, (ii) phase partitioning, i.e. air, water or lipid, or (iii) viscosity effects (Rothe 1997). Binding can either be reversible or irreversible depending on the strength of the interaction. Ketones and aldehydes were suggested to covalently bind with amino groups of proteins (Damodaran and Kinsella 1980; Weerawatanakorn et al. 2015). Others have shown that they can form weak hydrogen bonds with macromolecules containing electronegative clusters of nitrogen, sulphur or oxygen (Reineccius 2006; Tromelin et al. 2006). Although, there is no established mechanism of interaction between proteins and different classes of aroma compounds, previous studies have suggested covalent interactions between lysine and histidine residues of proteins and monounsaturated aldehydes and ketones such as hexanal, t-2-hexenal and butanone (Kikugawa et al. 1988; Meynier et al. 2004).

Mucins are the glycoprotein building blocks of the protective layer covering the mucosal membrane, lining the alimentary canal from the oral and nasal cavities through to the large intestine. They vary considerably in size, from a few thousand to several million daltons. The protein domains are rich in threonine and serine which form a bridge between their hydroxyl groups and the $\mathrm{N}$-acetylgalactosamine residues of the carbohydrate region. While most mucins are heavily glycosylated (up to 90\%), the 'naked' unglycosylated polypeptide is predominantly composed of cysteine, serine, threonine, lysine and proline which assist in the coiling of the glycoprotein which is thought to give rise to potential interaction sites with hydrophilic aroma compounds (Harding et al. 1983). These exposed amino groups are theoretically able to form several kinds of reactions with carbonyl groups, including Shiff bases or Michael addition reactions. These have also been demonstrated for more complex aldehyde compounds such as vanillin, shown to form Schiff bases with different amino acids including cysteine, lysine and phenylalanine (Kikugawa et al. 1988; Meynier et al. 2004; Ziegler 2007). In addition, reactions with cysteine were shown to be reversible under heat and acidic conditions, such as the stomach environment.

While low concentrations of aroma compounds may have negligible physical and chemical effects on the oral and intestinal mucus during oral processing, the very high concentrations of carbonyl containing flavour compounds inhaled by vaping are suggested to contribute significantly to the physical and chemical properties of the lung mucus, including viscosity effects and subsequent effects on the respiratory function. In light of this hypothesis, matrix free biophysical characterisation techniques of analytical ultracentrifugation (SV-AUC), viscometry and size exclusion chromatography multi angle laser light scattering (SECMALS) were employed to study some of the effects of model volatile compounds: linear aldehydes (hexanal, octanal, decanal) and linear ketones (butanone, hexanone, octanone, decanone) (Table 1). In addition, the effects of phenolic compounds (guaiacol, p-cresol and m-cresol) on the solution properties of bovine submaxillary mucin were probed using sedimentation velocity. This work along with future investigations will provide some of the starting points on the understanding some of the effects of high concentration 
Table 1 Lipophilicity $(\log P)$ and chemical representations of the volatile aroma compounds used in the bovine submaxillary mucin (BSM) interaction experiments

\begin{tabular}{cccc}
\hline Compound & LogP & Ketones & 0.37 \\
butanone & 1.38 \\
hexanone & 2.37 \\
octanone & 3.73 \\
decanone & Aldehydes \\
Phenols & 1.78 \\
octanal & 2.54 \\
decanal & 3.9 \\
guaiacol & 1.94 \\
p-cresol & 1.96 \\
m-cresol &
\end{tabular}

volatile aroma compounds on the respiratory but also on the gastro-intestinal system.

\section{Materials and methods}

\section{Sample preparation}

Bovine submaxillary mucin (BSM) (type I-S, M3895) and the volatile compounds were from Sigma-Aldrich (Dorset, UK). The experiments were prepared in $0.1 \mathrm{M}$ phosphate buffered saline (PBS), according to (Green 1933). Aldehydes and ketones were pre-solubilized in $70 \%$ ethanol by adding $1 \mathrm{~mL}$ aroma compound to $4 \mathrm{~mL} 70 \%$ ethanol to obtain a $20 \%$ (250 mg/mL stock solution). To prepare a $1 \mathrm{mg} / \mathrm{mL}$ solution of volatile compound, a $5 \mu \mathrm{L}$ aroma compound was added to a $955 \mu \mathrm{L}$ solution containing solubilized BSM. Phenols were added directly to their respective mucin solution.

\section{Analytical ultracentrifugation sedimentation velocity (SV)}

The experiments were performed at $20.0^{\circ} \mathrm{C}$ using the Rayleigh interference optical system in the Optima XL-I analytical ultracentrifuge (Beckman, Palo Alto, USA). A volume of $395 \mu \mathrm{L}$ sample and $405 \mu \mathrm{l}$ solvent, respectively, were injected into $12 \mathrm{~mm}$ double sector epoxy cells with sapphire windows, optically aligned to $0^{\circ}$ as described previously
(Channell et al. 2018). The samples were centrifuged at $30000 \mathrm{rpm}$ for $12 \mathrm{~h}$. Raw data were analysed in SEDFIT V16.1c using the least-squares boundary modelling $1 \mathrm{~s}-g^{*}(\mathrm{~s})$ method, abbreviated as $g(s)$ in this study, by generating sedimentation coefficient distributions, where $s$ is the rate of particle sedimentation or sedimentation coefficient (in Svedberg units, $\left.\mathrm{S}=10^{-13} \mathrm{~s}\right)$. The coefficient values from the $g(\mathrm{~s})$ vs s distributions were normalised to standard conditions (viscosity and density of solvent at $20.0{ }^{\circ} \mathrm{C}$ ) to give $\mathrm{s}_{20, w}$. A partial specific volume of $0.64 \mathrm{~mL} / \mathrm{g}$ was employed for mucin (Dodd et al. 1998; Fisher et al. 2006). TI (time invariant) and RI (radial invariant) noise were removed during data fitting. The distributions were exported and plotted in Origin 7.5 (Origin Lab, MA, US).

\section{Size exclusion chromatography: multi angle light scattering (SEC-MALS)}

The SEC set-up consisted of a Postnova Analysis PN7505 degassing unit (Landsberg am Lech Germany), Shimadzu LC-10AD HPLC Pump (Shimadzu UK, Milton Keynes, UK.), fitted with a Spark-Holland Marathon Basic autosampler (Spark Holland, Emmen, The Netherlands) combined with a TSK Gel guard column $(7.5 \times 75 \mathrm{~mm})$ and TSK Gel G5000, G6000 columns $(7.5 \times 300 \mathrm{~mm})$ connected in series (Tosoh Biosciences, Tokyo, Japan), fully flushed of column debris. Light scattering intensities were simultaneously detected at 14 angles as a function of elution volume using 
a DAWN ${ }^{\circledR}$ HELEOS $^{\mathrm{TM}}$ II, light scattering photometer connected in series to a ViscoStar ${ }^{\circledR}$ II on-line differential viscometer, an Optilab ${ }^{\circledR}$ rEX refractive index detector (Wyatt Technology Corporation, California, U.S.A.). A stock solution of $1.0 \mathrm{mg} / \mathrm{mL}$ BSM and BSM-hexanal was filtered through a $0.45 \mu \mathrm{m}$ syringe filter (Whatman, Maidstone, England) to remove any insoluble material or dust prior to injection and then injected into the autosampler. A $100 \mu \mathrm{l}$ aliquot of BSM and BSM-hexanal were injected onto the columns at ambient temperature $\left(20 \pm 3{ }^{\circ} \mathrm{C}\right)$ at a flow rate of $0.8 \mathrm{~mL} / \mathrm{min}$. ASTRA ${ }^{\mathrm{TM}}$ (Version 6) software (Wyatt Technology Corporation, Santa Barbara, U.S.A.) was used to estimate the weight average molecular weight, $M \mathrm{w}$ and radius of gyration $R_{\mathrm{g}}$, as a function of elution volume. The $4 \mathrm{~mW}$ $\mathrm{He}-\mathrm{Ne}$ laser was used at a wavelength of $632.8 \mathrm{~nm}$, and the refractive increment used was $0.181 \mathrm{~mL} / \mathrm{g}$. Because of the low solute concentrations and column dilution, non-ideality effects were assumed negligible.

\section{U-tube capillary (Ostwald) viscometry}

Relative viscosity was measured using the semi-automated (Schott Geräte, Hofheim, Germany) U-tube Ostwald capillary viscometer immersed in a temperature-controlled water bath at $20.00^{\circ} \mathrm{C}$. A constant volume of $2.0 \mathrm{~mL}$ was used for sampling BSM-hexanal mixture, automatically recorded six times. BSM concentrations were constant at $1.0 \mathrm{mg} / \mathrm{mL}$ while the buffer solution was made using different concentrations of hexanal. The intrinsic viscosity, $[\eta]$ was calculated according to the Solomon-Ciuta equation (Solomon and Ciuta 2019) at a constant BSM concentration " $c$ " of $1.0 \mathrm{mg} / \mathrm{mL}$ and plotted against the concentration of the solvent in Fig. 2 using:

$[\eta] \cong \frac{1}{c}\left(2\left(\eta_{s p}\right)-2 \ln \left(\eta_{r}\right)\right)^{1 / 2}$.

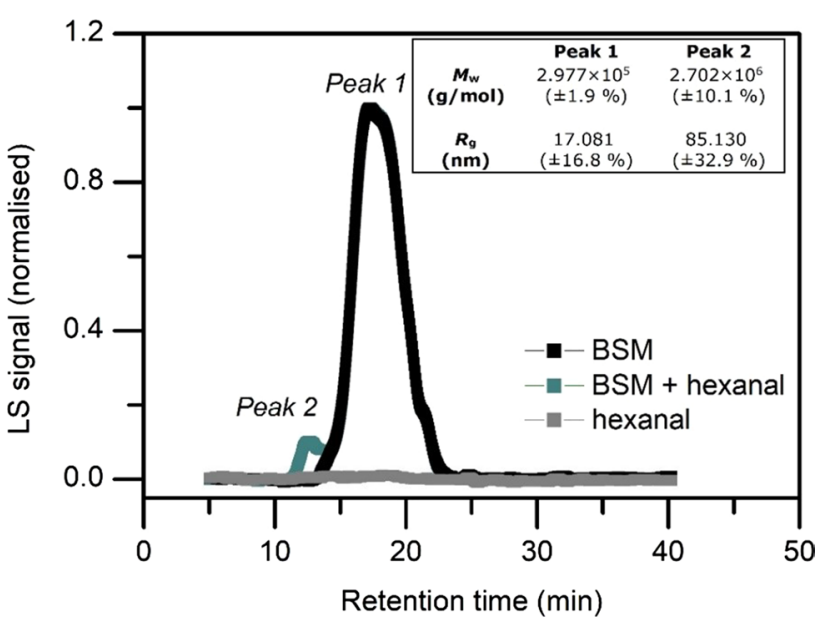

Fig. 2 SEC-MALS results showing the light scattering (LS) elution profile of BSM, hexanal and the result of their interaction. Insert shows the summary for the hydrodynamic parameters for the main peaks, such as the apparent weight average molar mass $\left(M_{\mathrm{w}}\right)$ and radius of gyration $\left(\mathrm{R}_{\mathrm{g}}\right)$
Fig. 1 Sedimentation velocity, $g(\mathrm{~s})$ analysis showing the sedimentation coefficient distributions of bovine submaxillary mucin $(1.0 \mathrm{mg} / \mathrm{mL})$ and the effect of aldehydes and ketones $(1.0 \mathrm{mg} / \mathrm{mL})$; and bottom: plot of hydrophobicity, $\log P$ against $\%$ change in mucin complexation as determined by the area under the normalised sedimentation coefficient curve. Rotor speed: 30,000 rpm (90,000 g), $20.0^{\circ} \mathrm{C}$
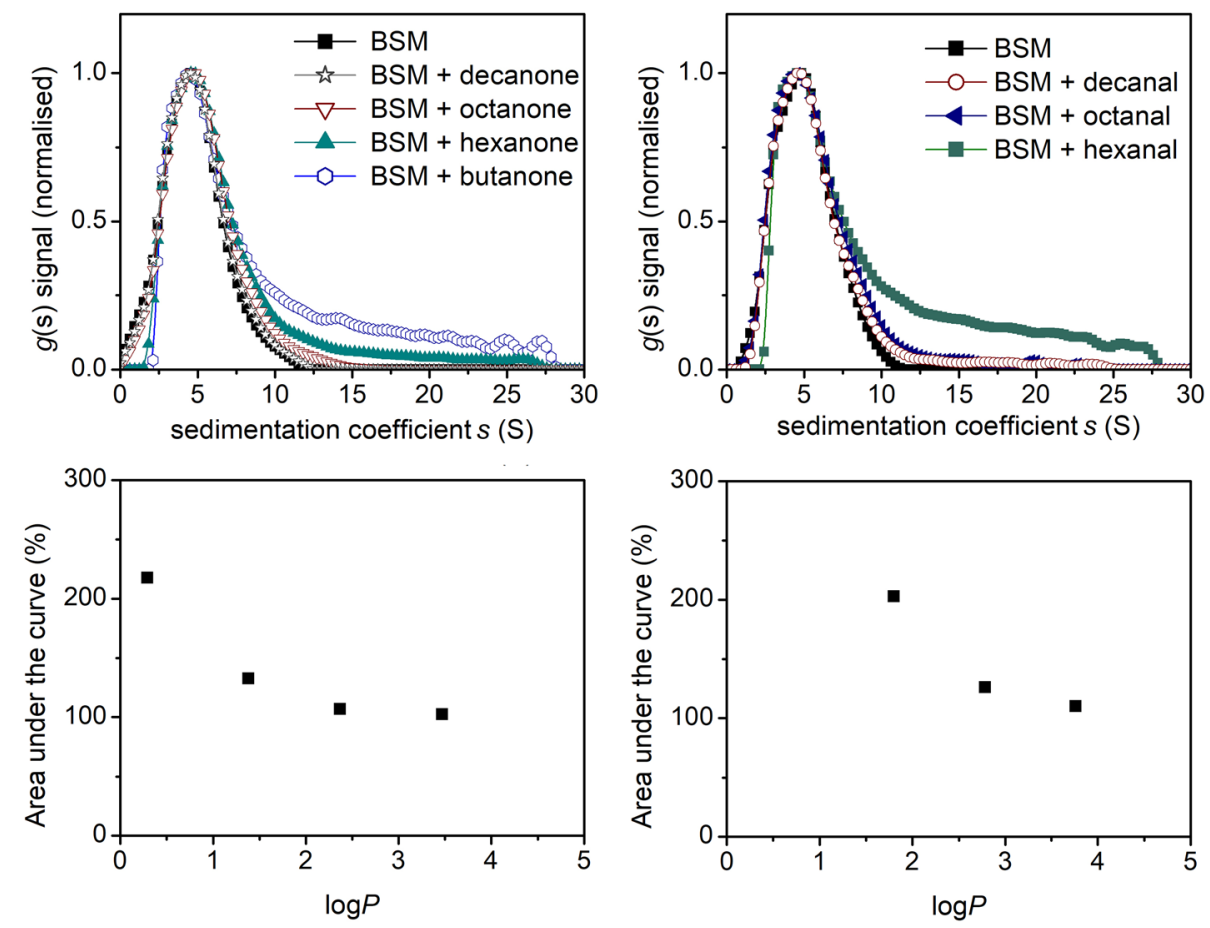


\section{Results and discussion}

\section{Carbonyl containing compounds}

The sedimentation velocity results show the distribution of sedimentation coefficients for $1.0 \mathrm{mg} / \mathrm{mL}$ BSM solution, revealing a broad polydisperse distribution ranging from 2 to $12 \mathrm{~S}$, as shown previously (Dinu et al. 2019a). The addition of linear aldehydes and ketone volatile compounds was shown to result in an increase in the proportion of species of high sedimentation rates, tailing up to $\sim 28 \mathrm{~S}$ for the mixtures containing more hydrophilic compounds such as butanone or hexanal (Fig. 1). The effect is negatively correlated with compound hydrophobicity, appearing milder for less soluble, longer chain aldehydes but also for ketones as a whole, possibly due to the alkyl group reducing their reactivity (see Table 1). In other words, the SV data suggest that the mucin interaction with the aldehydes and ketones is stronger for the shorter chain compounds, given their higher solubility in water. This can also be evidenced directly from the raw data (Appendix Fig. 6) in which changes in the fringe concentration and shape correspond to species of higher sedimentation coefficient and higher diffusion. Although there is some sedimentation contribution from the aroma compounds, in particular hexanal and butanone, being the most soluble (Appendix Fig. 7), the signal and apparent sedimentation coefficient are too low to suggest the possibility of concomitant sedimentation with the much larger mucin components.

The exact mechanism behind the increase in the higher $\mathrm{S}$ species remains unclear; however, AUC are indicative of non-specific mucin-solvent interactions causing either (i) partial mucin aggregation or (ii) changes in mucin conformation. In addition to the sedimentation results, an additional experiment was employed using the size exclusion chromatography coupled to a multi angle laser light scattering system (SEC-MALS) looking at the interaction of mucin with hexanal (Fig. 2).

During size exclusion chromatography, larger particles elute first, followed by smaller molecules. If they are too small to be detected by the column, as is the case of hexanal, they are not separated by the column and, therefore, no signal is recorded. The elution profiles (Fig. 2) reveal a broad multi-component distribution of BSM, ranging from $\sim 16$ to $24 \mathrm{~min}$, as reported previously (Dinu et al. 2019a). However, the addition of hexanal led to the formation of larger moieties (Peak 2), which appear to be up to ten times larger than the average molecular mass for Peak 1 . The apparent hydrodynamic radius $R_{\mathrm{g}}$ of the new complexes is $85 \mathrm{~nm}$, which is five times larger than the average $R_{\mathrm{g}}$ values for Peak 1 although the relatively high experimental errors indicates a certain degree of size heterogeneity in addition to the limited signal resulting from its small concentration, relative to the main peak.

To further examine the effect of the aldehyde containing solvent, the intrinsic viscosity of BSM was determined in the presence of different concentrations of hexanal. They were obtained via the Solomon-Ciuta equation and plotted against concentrations of hexanal (Fig. 3). An increase in the concentrations of hexanal led to an increase in the intrinsic viscosity of mucin, gradually plateauing at higher hexanal concentrations, which is attributed to the solubility of hexanal in water $\left(\sim 2.5 \mathrm{mg} / \mathrm{mL}\right.$ at $\left.20.0{ }^{\circ} \mathrm{C}\right)$. Currently, sedimentation, size exclusion and intrinsic viscosity results confirm an interaction between volatile aldehydes/ ketones and mucin. Although non-specific interactions between the glycoproteins and the solvent are suspected, due to an increase in the aggregation state of mucin, the sedimentation of the more hydrophilic aldehydes and ketones with the higher $\mathrm{S}$ species of the mucin is plausible, given the increase in the solute concentration (area under the curve). But the small increase in mucins intrinsic viscosity is consistent with small changes in conformation, which is suggestion to result from the binding of the carbonyls to amino regions of mucin, rather than solvent effects. A good way of confirming the complexation with the small solutes would be the use of gel chromatography, but previous attempts on mucin glycoproteins have proved unsuccessful due to smearing and inconsistent separation due to the very high molecular weight, high polydispersity and high degree of glycosylation.

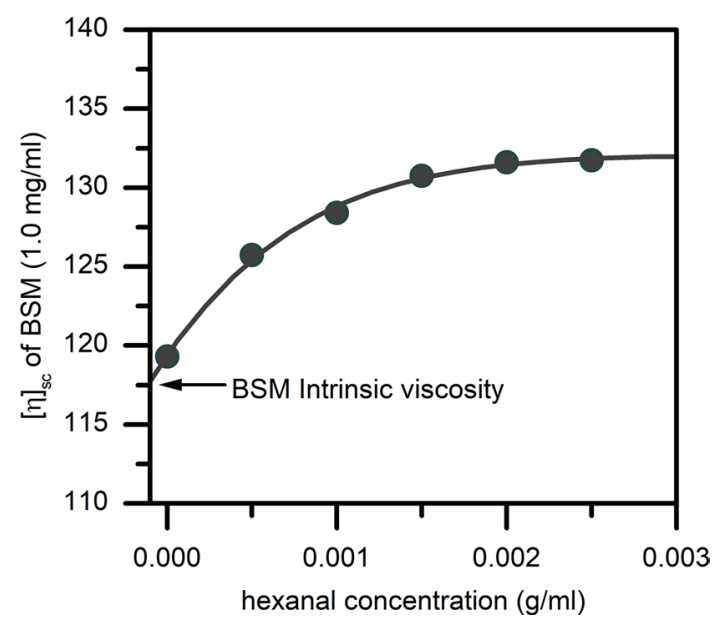

Fig. 3 Solomon-Ciuta intrinsic viscosity $[\eta]_{s c}$ analysis showing the quantitative effect of hexanal addition to bovine submaxillary mucin $(1.0 \mathrm{mg} / \mathrm{mL})$ in $0.1 \mathrm{M}$ phosphate buffer saline. The polynomial fit is based on six data points derived from the separate hexanal/BSM mixtures 


\section{Phenolic aroma compounds}

Next, the sedimentation velocity analysis was applied to study the effects of another class of bioactive volatile aroma compounds-phenols, which have also been found to elicit changes in the sedimentation properties of mucin (Fig. 4). Although they are not directly used as food flavourings, they can be found in a range of medicines or as bi-products generated during heating of e-cigarette aerosol (Dinu et al. 2020). Changes in the sedimentation coefficient of BSM upon the addition of guaiacol, p-cresol and $\mathrm{m}$-cresol suggest the formation of a smaller mucin components with a lower sedimentation coefficient, suggesting that phenols act as some type of molecular chaotropes.

These interactions were also studied by Raman spectroscopy, by examining changes in the vibrational spectrum of BSM before and after the addition of p-cresol (Appendix Fig. 8, Dinu et al. 2019a). Spectral changes were observed in the $820-850 \mathrm{~cm}^{-1}$ region, which represents a Fermi doublet, distinguished by the stronger $\left(840 \mathrm{~cm}^{-1}\right)$ and weaker $\left(810 \mathrm{~cm}^{-1}\right)$ Raman shifts. The ratio of the doublet is said to provide information on the strength of the hydrogen bonding of the phenoxy group in solution. Second, a decrease in the intensity of the $1500-1700 \mathrm{~cm}^{-1}$ Amide I and II region was observed, indicating changes in the non-glycosylated protein region of mucins (Dinu et al. 2019a).

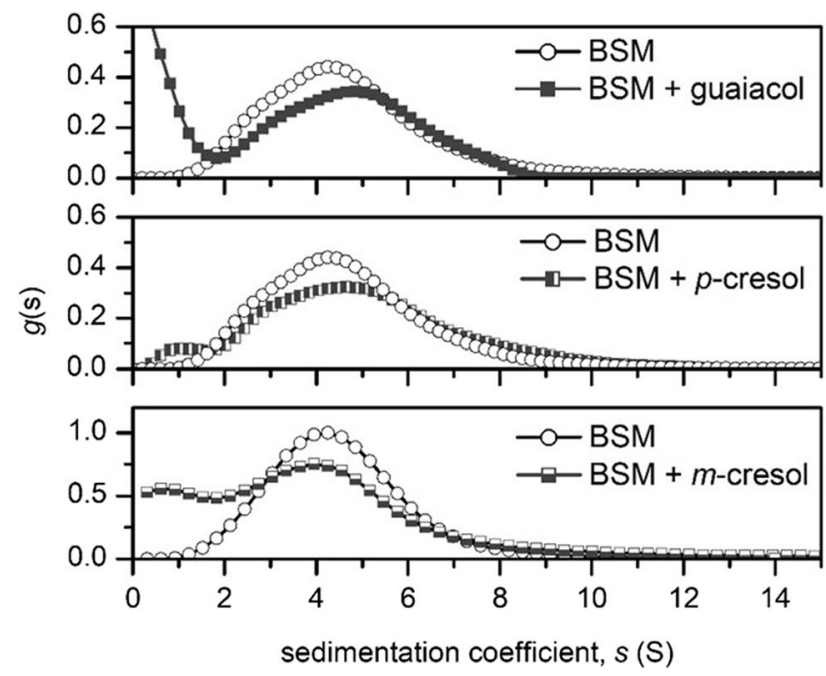

Fig. 4 Sedimentation velocity, $g(\mathrm{~s})$ analysis showing the sedimentation coefficient distributions of BSM $(0.5 \mathrm{~g} / \mathrm{mL})$ and the result of its interactions with different phenol volatile compounds $(0.5 \mathrm{mg} / \mathrm{mL})$. Rotor speed: $30,000 \mathrm{rpm}(90,000 \mathrm{~g}), 20.0^{\circ} \mathrm{C}$

\section{Discussion}

Protein-aroma interactions have been reported since the 1970's first study of protein-flavor binding (Arai et al. 1970). Using simple linear aldehydes and ketones, we aimed to gain some information on the reported interactions between flavourings which have a carbonyl functional group. SV-AUC was used to confirm that the interactions are dependent on the solubility of aldehyde and ketone flavours in water. Second, we have confirmed that aldehydes are more reactive than ketones, as shown by the increase in the concentrations and the rate of sedimenting boundaries (Appendix Fig. 6).

Other studies also confirmed a stronger affinity of aldehydes for binding of bovine serum albumin than ketones (Damodaran and Kinsella 1980). The mechanism of interaction is suggestion to arise from the binding between lysine residues and the carbonyl group of the monounsaturated aroma compounds (Kikugawa et al. 1988; Meynier et al. 2004). In addition, shorter more hydrophilic molecules such as acetaldehyde were previously demonstrated to increase the risk of developing alcoholic liver disease and hepatocellular carcinoma through a similar type of protein adduct formations, leading to impairment in protein function (Donohue et al. 1983). More recently, aroma-mucin interactions have also been proposed for more complex molecules, because there is a significant reduction in the gas phase concentration of octanal, nonanal and decanal in the presence of mucin at neutral $\mathrm{pH}$, reported using gas chromatography, reinforcing the hypothesis of aroma-mucin interactions (Dinu et al. 2019b). In other studies, vanillin and ethyl vanillin which also contain additional hydroxyl and ether groups were reported to interact with DNA at the A-T groove region under physiological conditions (Qais et al. 2019). Other work also found the use of vanillin as a DNA-dependent protein kinase inhibitor for use in anti-cancer therapies (Durant and Karran 2003). While the exact mechanism remains a topic of further investigation, the current SV, viscosity and size exclusion analysis suggests that the interactions induced by the small carbonyl containing compounds are inducing changes in the conformation and/or mucin aggregation.

Unlike aldehydes and ketones, some phenol molecules such as cresols and guaiacol, the latter being a phenol with a methoxy group, were found to exhibit the opposite effect. The sedimentation experiments indicate the presence of smaller components and a decrease in the mucin components. The additional Raman experiment shows a loss in the amide region, indicative of changes in the mucin region which is free of glycosylation. Previously, phenols such as $\mathrm{m}$-cresol have long been used as an excipient in insulin formulations to de-aggregate insulin and keep the proteins in their active, monomeric form (Whittingham et al. 1998). 
Fig. 5 Suggested effects on mucin-mucin interactions in solution: a Polysdisperse, random coil model of mucin in solution showing its multimeric assembly containing the glycosylated "bottlebrush" polypeptide backbone and the non-glycosylated "naked" protein region; b A proposed representation of the effect of chaotropic compounds on the solution structure of mucins; $\mathbf{c}$ Proposed representation of the effects of compounds causing mucin aggregation

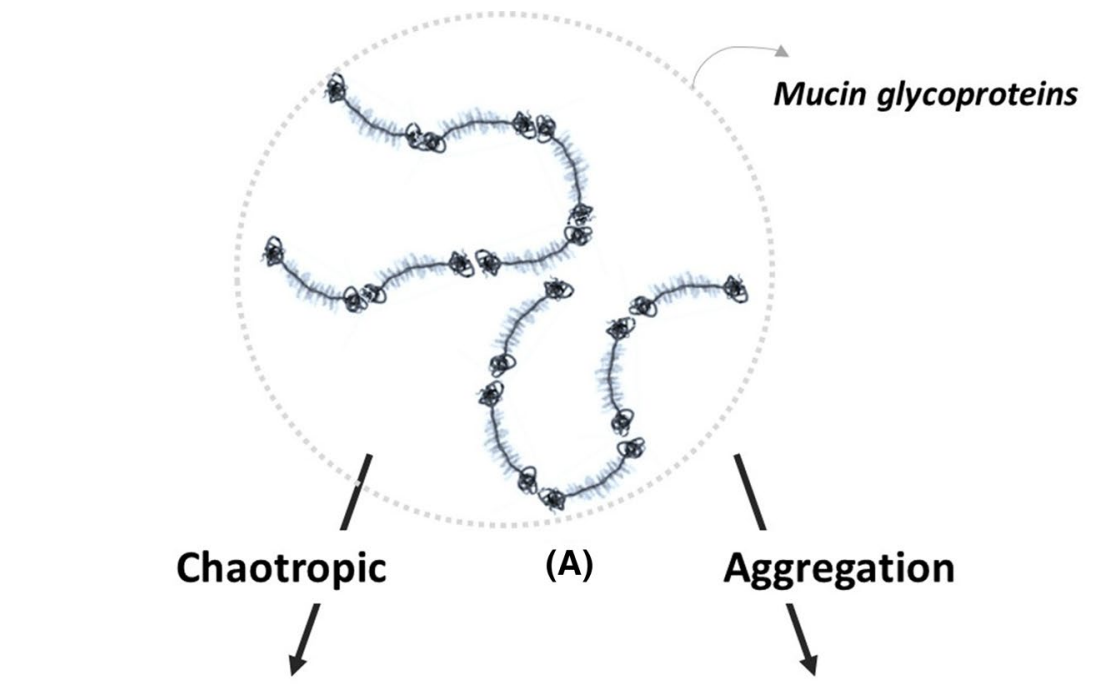

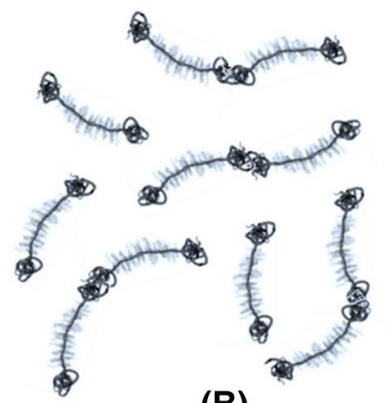

(B)

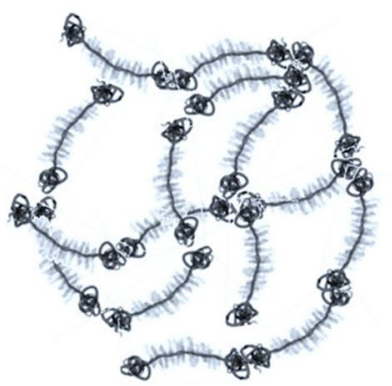

(C)
Guaiacol is another related compound which is used in the synthesis of other aroma compounds but also naturally present in the flavour of whiskey. It is used as a universal substrate for peroxidase enzymes, through the interaction with glycine and isoleucine with its phenoxy group (Murphy et al. 2012). It is worth suggesting that guaifenesin, which has the same functional groups as guaiacol, has been considered for reducing the molecular weight and viscosity of mucus in medication for patients suffering from mucus congestion issues caused by common colds (Seagrave et al. 2011). Previous work suggested that the hydroxyl group of the phenol ring (phenoxy group), is primarily involved in some type of interaction with mucin glycoproteins (Seagrave et al. 2011).

\section{Further remarks}

The exact nature by which carbonyl containing volatile compounds or the volatiles bearing a phenol group are not yet understood. Mucin glycoproteins are heavily dominated by charged oligosaccharides structures, which raise the possibility of electrostatic interactions events, such as charge shielding or charge repulsion, depending on the polarity of the volatile. Aldehydes and ketones are, therefore, suggested to have an effect on the viscoelastic properties of the mucus. In addition, volatiles bearing a phenol group are suggested to alter mucin-mucin interactions which are an important determinant in the properties of the mucus gel protecting the mucosal surfaces (Verdugo 2012). The dynamics by which the mucin stability is affected also appears to resemble that of chaotropic agents and detergents, such as urea or guanidinium hydrochloride $(\mathrm{GuHCl})$, which are known to disrupt non-covalent interactions (Radicioni et al. 2016). Based on the current data and previous studies, we, therefore, suggest two possible effects on mucin-mucin interactions (Fig. 5).

While the low concentrations of volatile aroma compounds in food may not elicit a significant change on the macrostructure of mucus glycoproteins, high concentrations are, therefore, suggested to lead to a significant impairment of mucosal function. If such high concentrations are inhaled, such as vaping products, this may also be relevant to the onset cytotoxic events reported in recent literature (Tierney et al. 2016; Omaiye 2019). Although, the components used in the current study are not desirable for use in e-cigarettes, they were used to understand the effect of more complex food flavour bearing the same types of functional groups, such as aldehydes, ketones or phenols. The two effects by which aroma compounds can affect the inherent solution properties of mucins are, therefore, proposed in the current hydrodynamic study (Fig. 5). 


\section{Conclusion}

This study demonstrates the evidence to suggest aroma compounds (aldehydes, ketones, and phenols) interacts with submaxillary mucin. Based on the sedimentation results, aldehydes appear to be more reactive than ketones, with shorter chain compounds leading to species of higher sedimentation coefficients. According to size exclusion chromatography and viscosity data for hexanal, it was shown that the interactions lead to species of higher apparent molar mass, size and higher intrinsic viscosity. It is unclear whether these effects are a consequence of a direct aroma-mucin complexation induced by the interaction between carbonyls and amino groups; however, larger particle formation and conformational changes are indicated. Further investigations will continue to analyze the nature of aroma-protein interactions.

In addition, SV-AUC indicated that phenols, such as guaiacol, p-cresol, m-cresol affect the sedimentation properties of mucin as evidenced from an increase in the concentration of smaller S fractions. Complemented by Raman spectroscopy, these changes appear to be related to the observed decrease in the amide I region $\left(\sim 1640 \mathrm{~cm}^{-1}\right)$, suggesting interactions are occurring in the non-glycosylated protein region of mucins. Future studies will focus on the analysis of proteins which have a well-defined structure to investigate the chemical and physical mechanisms of aroma-protein interactions. For instance, the use of the multi-wavelength detector capability could allow the screening of multiple aroma compounds with different optical properties at once, in the presence of heterogeneous mixtures of mucosal proteins and glycoproteins.
Acknowledgements This work was supported by the Engineering and Physical Sciences Research Council [Grant number EP/L015633/1], Biotechnology and Biological Sciences Research Council [Grant number BB/T006404/1] and the 2019 EPSRC Doctoral Prize fellowship. The work was carried out at the National Centre for Macromolecular Hydrodynamics, in the School of Biosciences, University of Nottingham.

Author contributions V.D. performed the experiments and wrote the manuscript. T.M. performed the SEC-MALS experiments and data interpretation. G.E.Y. wrote and commented on the manuscript. S.E.H. and I.F. supervised and commented on the manuscript.

Data availability Data are available upon reasonable request.

\section{Compliance with ethical standards}

Competing interest The authors declare that they have no known competing financial interests or personal relationships that could have appeared to influence the work reported in this paper.

Open Access This article is licensed under a Creative Commons Attribution 4.0 International License, which permits use, sharing, adaptation, distribution and reproduction in any medium or format, as long as you give appropriate credit to the original author(s) and the source, provide a link to the Creative Commons licence, and indicate if changes were made. The images or other third party material in this article are included in the article's Creative Commons licence, unless indicated otherwise in a credit line to the material. If material is not included in the article's Creative Commons licence and your intended use is not permitted by statutory regulation or exceeds the permitted use, you will need to obtain permission directly from the copyright holder. To view a copy of this licence, visit http://creativecommons.org/licenses/by/4.0/.

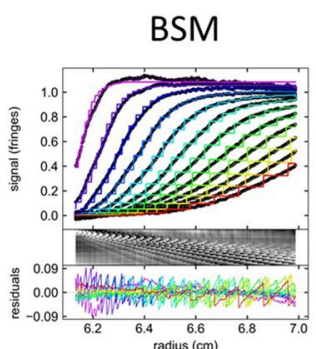

BSM

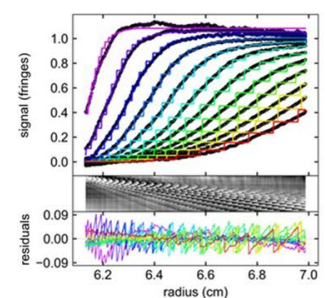

+ decanone

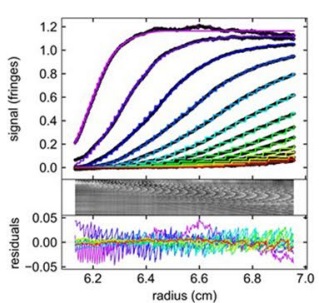

+ decanal

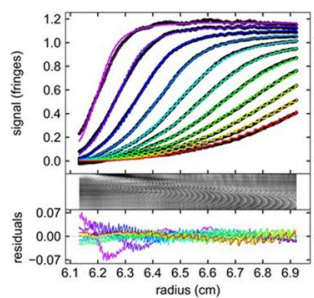

+ octanone

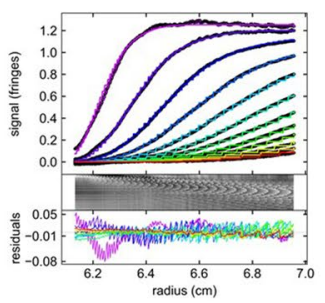

+ octanal

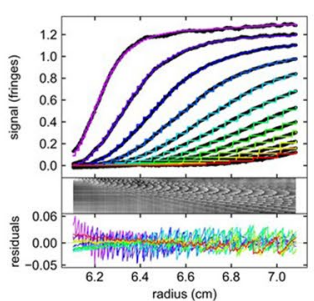

+ hexanone

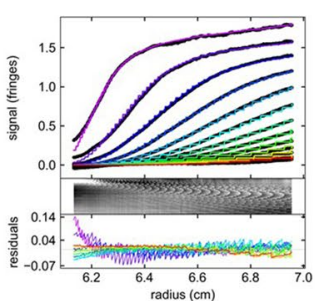

+ hexanal

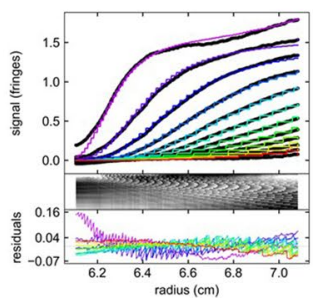

+ butanone

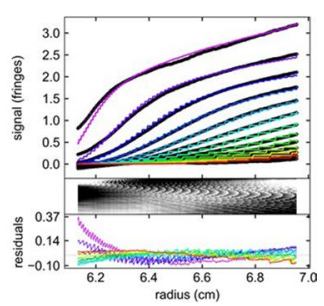

hexanal only

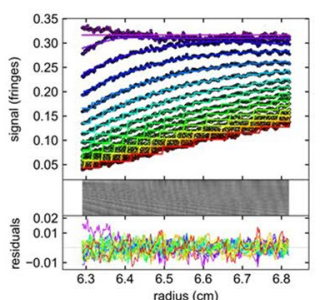

Fig. 6 Raw data showing the changes in fringe concentration and residuals plots for BSM, BSM/aroma compounds mixtures and hexanal control, plotted using the GUSSI extension in SEDFIT (Brautigam 2015) 


\section{Appendix}

See Figs. 6, 7 and 8.

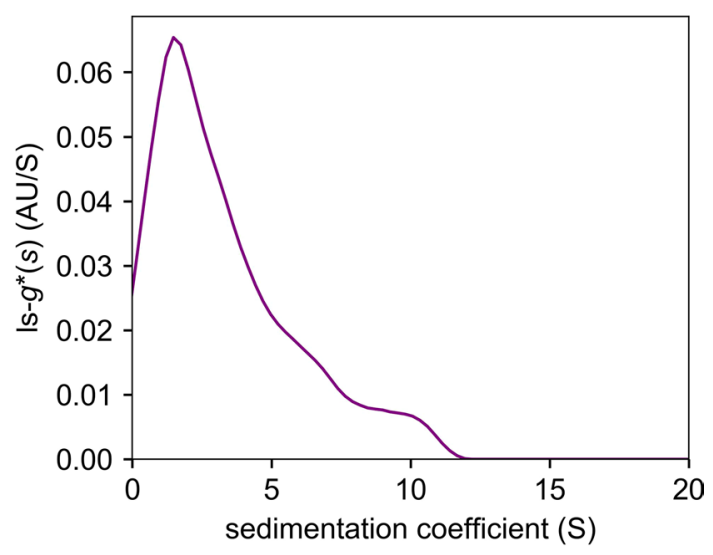

Fig. 7 Sedimentation coefficient distribution of hexanal control $(1.0 \mathrm{mg} / \mathrm{mL})$ in $0.1 \mathrm{M}$ PBS pH 7. Rotor speed: $30,000 \mathrm{rpm}$ $(90,000 \mathrm{~g}), 20.0^{\circ} \mathrm{C}$

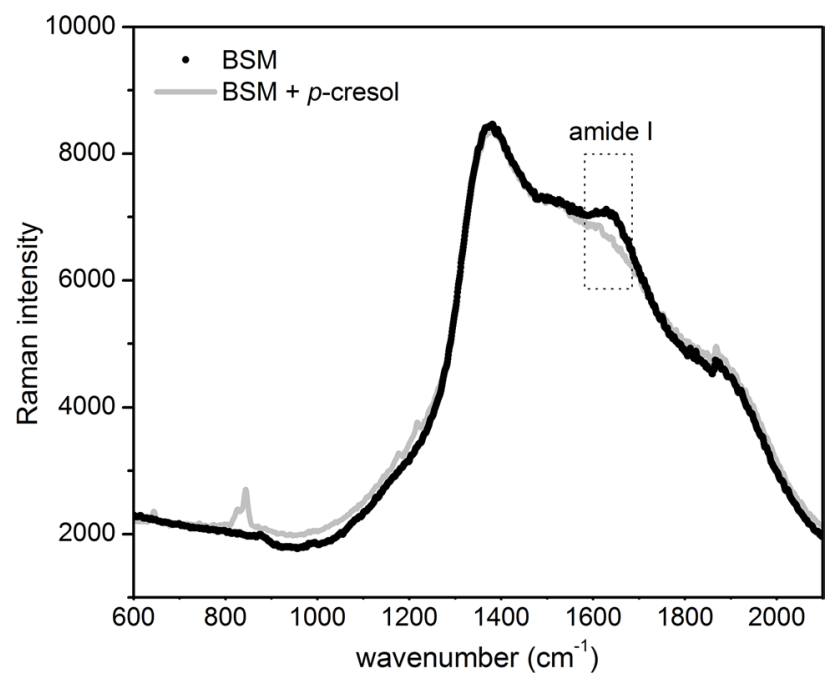

Fig. 8 Raman spectra of BSM $(c=10 \mathrm{mg} / \mathrm{mL})$ with and without p-cresol. The mucin/p-cresol sample was dialysed against a $14 \mathrm{kDa}$ membrane prior to Raman analysis to remove excess p-cresol. Performed in 0.1 M PBS pH 7 at $20.0{ }^{\circ} \mathrm{C}$. Adapted from (Dinu et al. 2019a)

\section{References}

Arai S, Noguchi M, Yamashita M et al (1970) Studies on flavor components in soybean. Agric Biol Chem 34:1569-1573. https://doi. org/10.1271/bbb1961.34.1569
Brautigam CA (2015) Calculations and publication-quality illustrations for analytical ultracentrifugation data. Methods Enzymol 562:109-133. https://doi.org/10.1016/bs.mie.2015.05.001

Channell G, Dinu V, Adams GG, Harding SE (2018) A simple cellalignment protocol for sedimentation velocity analytical ultracentrifugation to complement mechanical and optical alignment procedures. Eur Biophys J 47:809-813. https://doi.org/10.1007/ s00249-018-1328-9

Cohen SM, Eisenbrand G, Fukushima S, et al (2020) GRAS 29 Flavoring Substances. https://www.ift.org/news-and-publications/foodtechnology-magazine/issues/2020/march/features/gras-29-flavo ring-substances. Accessed 2 Jul 2020

Damodaran S, Kinsella JE (1980) Flavor protein interactions. Binding of carbonyls to bovine serum albumin: thermodynamic and conformational effects. J Agric Food Chem 28:567-571. https:// doi.org/10.1021/jf60229a019

Dinu V, Gillis RB, MacCalman T et al (2019) Submaxillary mucin: its effect on aroma release from acidic drinks and new insight into the effect of aroma compounds on its macromolecular integrity. Food Biophys. https://doi.org/10.1007/s11483-019-09574-2

Dinu V, Kilic A, Wang Q et al (2020) Policy, toxicology and physicochemical considerations on the inhalation of high concentrations of food flavour. Npj Sci Food 4:15. https://doi.org/10.1038/s4153 8-020-00075-y

Dinu V, Yakubov G, Lim M et al (2019) Mucin immobilization in calcium alginate: a possible mucus mimetic tool for evaluating mucoadhesion and retention of flavour. Int J Biol Macromol. https ://doi.org/10.1016/j.ijbiomac.2019.07.148

Dodd S, Place GA, Hall RL, Harding SE (1998) Hydrodynamic properties of mucins secreted by primary cultures of Guinea-pig tracheal epithelial cells: determination of diffusion coefficients by analytical ultracentrifugation and kinetic analysis of mucus gel hydration and dissolution. Eur Biophys J 28:38-47. https://doi.org/10.1007/ s002490050181

Donohue TM, Tuma DJ, Sorrell MF (1983) Acetaldehyde adducts with proteins: binding of [14C] acetaldehyde to serum albumin. Arch Biochem Biophys 220:239-246

Durant S, Karran P (2003) Vanillins-a novel family of DNA-PK inhibitors. Nucleic Acids Res 31:5501-5512. https://doi.org/10.1093/ nar/gkg753

Fares K, Landy P, Guilard R, Voilley A (1998) Physicochemical interactions between aroma compounds and milk proteins: effect of water and protein modification. J Dairy Sci 81:82-91. https://doi. org/10.3168/jds.S0022-0302(98)75554-7

FEMA (2020a) Safety and regulatory authority to use flavors-focus on vaping products I FEMA. https://www.femaflavor.org/safety-regul atory-authority-use-flavors-focus-vaping-products. Accessed 2 Jul 2020

Fisher SZ, Govindasamy L, Tu C et al (2006) Structure of human salivary $\alpha$-amylase crystallized in a C-centered monoclinic space group. Acta Crystallograph Sect F Struct Biol Cryst Commun 62:88-93. https://doi.org/10.1107/S1744309105042491

Green AA (1933) The preparation of acetate and phosphate buffer solutions of known $\mathrm{pH}$ and ionic strength. J Am Chem Soc 55:23312336. https://doi.org/10.1021/ja01333a018

Harding SE, Rowe AJ, Creeth JM (1983) Further evidence for a flexible and highly expanded spheroidal model for mucus glycoproteins in solution. Biochem J 209:893-896. https://doi.org/10.1042/bj209 0893

Jouenne E, Crouzet J (2000) Effect of pH on retention of aroma compounds by beta-lactoglobulin. J Agric Food Chem 48:1273-1277

Kikugawa K, Iwata A, Beppu M (1988) Formation of cross-links and fluorescence in polylysine, soluble proteins and membrane proteins by reaction with 1-butanal. Chem Pharm Bull (Tokyo) 36:685-692. https://doi.org/10.1248/cpb.36.685 
Linden SK, Sutton P, Karlsson NG et al (2008) Mucins in the mucosal barrier to infection. Mucosal Immunol 1:183-197. https://doi. org/10.1038/mi.2008.5

Meynier A, Rampon V, Dalgalarrondo M, Genot C (2004) Hexanal and $\mathrm{t}$-2-hexenal form covalent bonds with whey proteins and sodium caseinate in aqueous solution. Int Dairy J 14:681-690. https://doi. org/10.1016/j.idairyj.2004.01.003

Murphy EJ, Metcalfe CL, Nnamchi C et al (2012) Crystal structure of guaiacol and phenol bound to a heme peroxidase. FEBS J 279:1632-1639. https://doi.org/10.1111/j.1742-4658.2011.08425 . $\mathrm{x}$

Omaiye EE (2019) High concentrations of flavor chemicals are present in electronic cigarette refill fluids. Sci Rep 9:1-9

Paravisini L, Guichard E (2016) Interactions between aroma compounds and food matrix. In: Flavour. John Wiley \& Sons, Ltd, pp 208-234. https://doi.org/10.1002/9781118929384

Parker JK, Elmore S, Methven L (2015) Flavour development, analysis and perception in food and beverages. Elsevier. https://doi. org/10.1016/C2013-0-16460-4

Qais FA, Husain FM, Khan MS (2019) Deciphering the interaction of food additive, vanillin with DNA: a biophysical and computational study. J Biomol Struct Dyn. https://doi.org/10.1080/07391 102.2019.1692693

Radicioni G, Cao R, Carpenter J et al (2016) The innate immune properties of airway mucosal surfaces are regulated by dynamic interactions between mucins and interacting proteins: the mucin interactome. Mucosal Immunol 9:1442-1454. https://doi.org/10.1038/ mi.2016.27

Reineccius G (2006) Flavor release from foods. Flavour chemistry and technology. CRC Press, Boca Raton, pp 139-159

Rothe M (1997) Flavor-food interactions. ACS symposium series 633. edited by R. J. McGorrin and J. V. Leland. XII and 235 pages, numerous figures and tables. American Chemical Society, Washington, DC, 1996. Price: 89.95 US \$. Food Nahr 41:379-379. https://doi.org/10.1002/food.19970410618
Seagrave J, Albrecht H, Park YS et al (2011) Effect of guaifenesin on mucin production, rheology, and mucociliary transport in differentiated human airway epithelial cells. Exp Lung Res 37:606-614. https://doi.org/10.3109/01902148.2011.623116

Solomon OF, Ciuta IZ (2019) Détermination de la viscosité intrinsèque de solutions de polymères par une simple détermination de la viscosité-Solomon-1962 — Journal of Applied Polymer Science-Wiley Online Library. https://onlinelibrary.wiley.com/ doi/abs/https://doi.org/10.1002/app.1962.070062414. Accessed 6 May 2019

Tierney PA, Karpinski CD, Brown JE et al (2016) Flavour chemicals in electronic cigarette fluids. Tob Control 25:10-15

Tromelin A, Andriot I, Guichard E (2006) Protein-flavour interactions. In: Flavour in Food. Woodhead Publishing Limited, p 320. https ://doi.org/10.1533/9781845691400.2.172

Verdugo P (2012) Supramolecular dynamics of mucus. Cold Spring Harb Perspect Med 2:a009597. https://doi.org/10.1101/cshperspec t.a009597

Weerawatanakorn M, Wu J-C, Pan M-H, Ho C-T (2015) Reactivity and stability of selected flavor compounds. J Food Drug Anal 23:176-190. https://doi.org/10.1016/j.jfda.2015.02.001

Whittingham JL, Edwards DJ, Antson AA et al (1998) Interactions of phenol and $\mathrm{m}$-cresol in the insulin hexamer, and their effect on the association properties of B28 pro-Asp insulin analogues. Biochemistry 37:11516-11523. https://doi.org/10.1021/bi980807s

Ziegler H (2007) Flavourings: Production, Composition, Applications, Regulations. John Wiley \& Sons. https://doi.org/10.1002/97835 27611454

Publisher's Note Springer Nature remains neutral with regard to jurisdictional claims in published maps and institutional affiliations. 